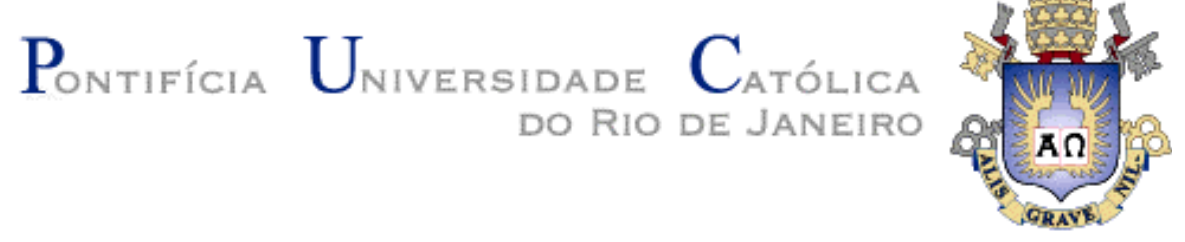

Olga Cecilia Carvajal García

Modelagem sísmica em arenitos: efeito da dispersão da velocidade e do tipo de fluido

TESE DE DOUTORADO

Departamento De Engenharia CiviL Programa de Pós-graduação em Engenharia Civil 


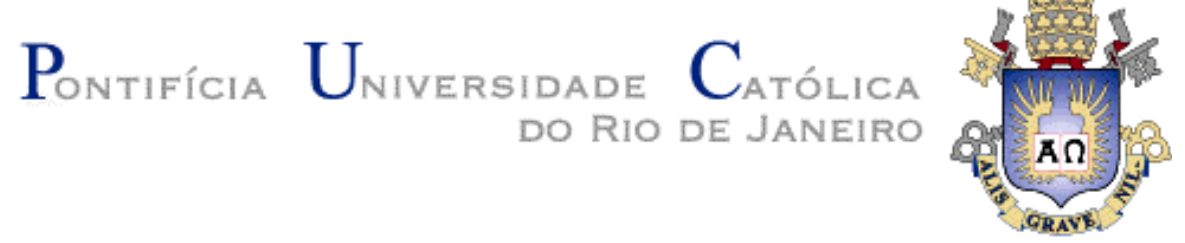

Olga Cecilia Carvajal García

\section{MODELAGEM SÍSMICA EM ARENITOS: EFEITO DA DISPERSÃO DA VELOCIDADE E DO TIPO DE FLUIDO}

Tese de Doutorado

Tese apresentada ao Programa de Pós-graduação em Engenharia Civil da PUC-Rio como requisito parcial para obtenção do título de Doutor em Engenharia Civil.

Orientador: Sérgio Augusto Barreto da Fontoura 


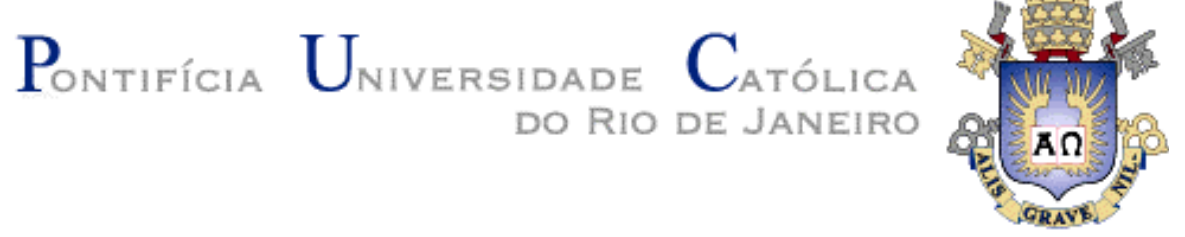

Olga Cecilia Carvajal García

\section{Modelagem Sísmica em Arenitos: Efeito da Dispersão da Velocidade e do Tipo de Fluido}

Tese apresentada como requisito parcial para obtenção do título de Doutor pelo Programa de Pós-Graduação em Engenharia Civil da PUC-Rio. Aprovada pela Comissão Examinadora abaixo assinada.

Prof. Sérgio Augusto Barreto da Fontoura Orientador

Departamento de Engenharia Civil - PUC-Rio

Prof. Raul Rosas e Silva Departamento de Engenharia Civil - PUC-Rio

Dr. Marco Antonio Cetale Santos Departamento de Engenharia Elétrica - PUC-Rio

Dr. Carlos Rodríguez Suárez PETROBRÁS

Dra . Cleide Jeane R. Bacelar Fundação GORCEIX / CENPES / PETROBRÁS

Prof. José Eugênio Leal Coordenador Setorial do Centro

Técnico Científico - PUC-Rio

Rio de Janeiro, 7 de Maio de 2008 
Todos os direitos reservados. É proibida a reprodução total ou parcial do trabalho sem autorização da universidade, do autor e do orientador.

\section{Olga Cecília Carvajal García}

Graduou-se em Engenharia Civil na UIS (Universidade Industrial de Santander - Colômbia) em 2000. Defendeu sua dissertação de Mestrado em Engenharia Civil (Geotecnia) na Pontifícia Universidade Católica do Rio de Janeiro (PUC-Rio) em 2003.

Ficha Catalográfica

García, Olga C. Carvajal

Modelagem sísmica em arenitos: efeito da dispersão da velocidade e do tipo de fluido / Olga C. Carvajal Garcia; orientador: Sérgio Augusto B. da Fontoura. - Rio de Janeiro: PUC, Departamento de Engenharia Civil, 2008.

167 f.: il. ; $29,7 \mathrm{~cm}$

Tese (doutorado) - Pontifícia Universidade Católica do Rio de Janeiro, Departamento de Engenharia Civil.

Inclui referências bibliográficas.

1. Engenharia Civil - Teses. 2. Modelagem sísmica. 3. Simulação de reservatórios. 4. Substituição de fluidos. 5. Fluxo local. 6. Upscaling. 7. Física de rochas. I. Fontoura, Sérgio Augusto B. da. II. Pontifícia Universidade Católica do Rio de Janeiro. Departamento de Engenharia Civil. III. Título. 


\section{Agradecimentos}

À ANP e à PUC-Rio pelo apoio financeiro concedido durante o meu doutorado.

Agradeço imensamente ao professor Sérgio A. B. da Fontoura pela oportunidade de desenvolvimento, pela sua amizade, confiança e respeito profissional depositados na minha pessoa. Obrigada pela estrutura oferecida através do GTEP, cujo apoio foi essencial.

A todos os professores e funcionários do Departamento de Engenharia Civil pelos ensinamentos transmitidos.

Aos colegas e amigos do GTEP. Agradeço especialmente à Nelly e ao Julio pela sua amizade e companheirismo.

Agradeço ao Vladimir e ao Fredy Artola pelas discussões sobre física de rochas e pela motivação para a execução deste trabalho. Agradeço especialmente ao Carlos Claudino, pela sua amizade, otimismo e importante contribuição neste trabalho.

Agradeço ao Julio pelos momentos compartilhados. Agradeço aos amigos Carolina e Fredy Angarita pelo apoio no começo deste caminho. Agradeço áqueles que de uma ou outra forma estiveram comigo, de perto ou de longe, nestes anos todos no Brazil.

Agradeço aos meus pais, Rafael e Cecilia, por tudo o que eles investiram na minha vida. Agradeço especialmente à minha mãe, e às minhas irmãs, Claudia e Sandra, por todo amor e confiança que a distância tornou ainda mais fortes.

Agradeço muito a Deus por todos os momentos que constituíram a minha experiência de vida aqui, e por me brindar a força para conseguir este propósito. 


\section{Resumo}

García, Olga C. Carvajal; Fontoura, S. A. B. Modelagem sísmica em arenitos: efeito da dispersão da velocidade e do tipo de fluido. Rio de Janeiro, 2008. 167p. Tese de Doutorado - Departamento de Engenharia Civil, Pontifícia Universidade Católica do Rio de Janeiro.

O conhecimento do que acontece no reservatório em produção a partir de variações temporais dos atributos sísmicos devido aos processos dinâmicos vem atingindo um valor crescente na indústria do petróleo, especialmente em arenitos. Este processo possui vários desafios, focados em grande parte a desvendar a superposição dos diferentes efeitos provocados pelas mudanças do reservatório nos dados sísmicos. As propriedades sísmicas são afetadas de maneira complexa por vários fatores, sendo a saturação um dos mais importantes, principalmente em rochas porosas como o arenito. Esta propriedade influencia no módulo elástico da rocha e sua resposta sísmica e, ao mesmo tempo, introduz dispersão da velocidade (variação da velocidade com a freqüência). A transição de fluido efetivo (distribuição homogênea e menores velocidades) para fluido com distribuição heterogênea (e maiores velocidades) estabelece um mecanismo de dispersão presente para freqüências sísmicas in situ, especialmente no arenito. O método mais utilizado para aplicar a técnica de substituição de fluidos se baseia na teoria de Gassmann (1951), que considera o meio poroso estático (estado de isostress), onde o fluido não é afetado pela perturbação da onda. No entanto, pesquisas mostram que as velocidades acústicas em rochas saturadas de fluido dependem da freqüência, do tipo de fluido e sua distribuição no meio poroso, viscosidade e outras propriedades que tornam as ondas dispersivas. Neste trabalho são realizadas simulações de fluxo de reservatórios, transformações de física de rochas, upscaling e modelagem sísmica em cenários de injeção de gás com o objetivo de esclarecer a importância de levar em conta a dispersão da velocidade na análise time-lapse. Para isso, são analisados para cada modelo mapas de saturação, velocidade, impedância e sismogramas sintéticos (seções de contraste) calculados com as teorias de substituição Gassmann (1951) e Mavko \& Jizba (1991). Os resultados mostram que a resposta sísmica pode ter um incremento de até $15 \%$ quando a dispersão devida ao fluxo local é considerada. Porosidade e tortuosidade são parâmetros essenciais que influenciam de maneira diferente na resposta sísmica. 


\section{Palavras-chave}

Modelagem sísmica; simulação de reservatórios; substituição de fluidos; fluxo local; upscaling; física de rochas. 


\section{Abstract}

García, Olga C. Carvajal; Fontoura, S. A. B. Sandstone seismic modeling: effects of velocity dispersion and fluid type. Rio de Janeiro, 2008, 167p. D.Sc. Thesis. Civil Engineering Departament, Pontifícia Universidade Católica do Rio de Janeiro.

The evaluation of reservoir dynamics during production through time-lapse interpretation has reached a substantial importance in the petroleum industry, mainly in sandstones. This evaluation presents many challenges, mainly concerned to unmask the overlapping of different effects in seismic data due to reservoir changes. Several factors affect seismic properties and saturation is one of the most important. This property influences the rock bulk modulus and seismic response and also causes a velocity dependence on the frequency. This phenomenon is known as velocity dispersion. Furthermore, the transition from effective homogeneous fluid to heterogeneous saturation represents a dispersion mechanism that appears for seismic frequencies in situ in sandstones. The most commonly method used to perform the fluid substitution technique is based in Gassmann theory (1951). This approach considers a static porous media (isostress condition), where fluid is not affected by wave propagation. However, it is well known that acoustic velocities in fluid saturated rocks depends on frequency, according to fluid type and distribution on porous media, viscosity, and others properties that become waves dispersive. In this work reservoir flow-simulation, rock physics transformations, upscaling and seismic modeling were performed in gas injection scenarios. Synthetic seismograms and some contrast sections were generated using Gassmann (1951) and Mavko \& Jizba (1991) substitution theories. The goal is to clarify the relevance of considering velocity dispersion on time-lapse seismic analyzing possible differences in the seismic parameters. Results show that seismic response could increase in $15 \%$ when squirt flow dispersion is considered. Porosity and tortuosity are essential parameters to analyze seismic response.

\section{Keywords}

Seismic modeling; reservoir simulation; fluid substitution; squirt flow; upscaling; rock physics. 


\section{Sumário}

1 Introdução 24

1.1. Descrição do Problema 24

1.2. Motivação 25

1.3. Objetivo 26

1.4. Descrição 27

2 Fundamentos Teóricos 29

2.1. Sísmica Time-Lapse $\quad 29$

2.2. Efeitos da Saturação na Velocidade 33

2.2.1. Efeito do Fluido 33

2.2.2. Efeito da Freqüência 38

2.3. Distribuição de Fluido e Resposta Sísmica 47

2.3.1. Modelo de Saturação Uniforme 50

2.3.2. Modelo de Saturação Heterogênea (Patchy) 52

2.4. Simulação de Fluxo e Modelagem Sísmica: Problema de Escala 54

3 Influência da Distribuição do Fluido na Variação da Velocidade Compressional $(V p) \quad 59$

3.1. Introdução $\quad 59$

3.2. A Escala como Fator de Dispersão da Velocidade 60

3.3. Efeito da Temperatura 61

3.4. Efeito da Pressão 66

3.5. Porosidade e Dispersão da Velocidade 68

3.6. Conclusão 71

4 Simulação de Fluxo em Reservatório Sintético 72

4.1. Modelo de Reservatório 73

4.1.1. Propriedades da Rocha 75

4.1.2. Propriedades dos Fluidos $\quad 75$

4.1.3. Esquema de Injeção 78 
4.2. Resultados $\quad 79$

4.2.1. Seções de Saturação 79

4.2.2. Seções de Pressão 82

5 Modelagem Sísmica no Tempo 85

5.1. Campos de Velocidade e Impedância Acústica 85

5.1.1. Modelos de Física de Rochas 86

5.1.2. Características do Modelo 88

5.1.3. Cálculo da Velocidade Compressional e Impedância Acústica 91

5.2. Seções Sísmicas 107

6 Conclusões e Sugestões 120

6.1. Conclusões 120

6.2. Sugestões 121

7 Referências Bibliográficas 123

APÊNDICE A Programas Utilizados 128

$\begin{array}{ll}\text { A.1 UTCHEM } & 128\end{array}$

$\begin{array}{ll}\text { A.2 TESSERAL } & 129\end{array}$

$\begin{array}{ll}\text { A.3 HYDRO_GEN } & 130\end{array}$

APÊNDICE B Rotinas MATLAB: Modelos de Saturação Homogêneo e Heterogêneo 131

B.1 Arenito Beaver - Efeito da Escala 131

B.2 Chert Monterrey - Efeito da Temperatura 133

B.2.1 Óleo Leve 133

B.2.2 Óleo Pesado 136

B.2.3 Óleo Leve versus Pesado 140

$\begin{array}{ll}\text { B.3 Areia Limpa - Efeito da Pressão } & 147\end{array}$

B.4 Areia Limpa e Areia Argilosa - Efeito da Porosidade 150

B.5 Arenito Fontainebleu 154 
APÊNDICE C Código MATLAB: Upscaling da Saturação Limite Homogêneo e Patchy Cálculo de $V_{p}$ e Impedância Acústica 159

C.1. Método de Gassmann: $V_{p}$ para escala fina e grossa 159

C.2. Método de Mavko \& Jizba: $V_{p}$ para escala fina e grossa 163 


\section{Lista de figuras}

Figura 2.1 - Princípio físico do monitoramento sísmico

(Calvert, 2005a).

Figura 2.2 - Exemplo de levantamento base, monitor e a diferença (Calvert, 2005b).

Figura 2.3 - Variação da impedância com porosidade (Lumley \& Behrens, 1998).

Figura 2.4 - Valores de $K_{o}$ obtidos com as equações de Voigt,

Reuss e Hill (Smith, 2003).

Figura 2.5 - Esquema do fluxo de Biot decorrente da propagação da onda através do meio poroso (modificado de Akbar, 1993).

Figura 2.6 - Dispersão da velocidade compressional de Biot em 122 amostras de arenito (Wang \& Nur, 1992).

Figura 2.7 - Esquema do fluxo local (squirt-flow) decorrente da propagação da onda através do meio poroso (modificado de Akbar, 1993).

Figura 2.8 - Atenuação sísmica como função da freqüência $x$ viscosidade para diferentes pressões efetivas e saturações (Jones, 1986).

Figura 2.9 - Freqüência característica no modelo de fluxo local (Wang, 2000).

Figura 2.10 - Variação do módulo bulk da rocha saturada $\left(\Delta K_{\text {rell }}\right)$ segundo as fissuras presentes no meio e a razão de achatamento, $\alpha$ (Endres \& Knight, 1997).

Figura 2.11 - Variação de $V_{p}$ em função da saturação para uma rocha submetida a ensaio de embebição e drenagem (Cadoret, 1993).

Figura 2.12 - Dependência da escala de difusão crítica da freqüência e da permeabilidade para uma rocha saturada de óleo com $\eta=5 \mathrm{cp}$.

Figura 2.13 - Esquema mostrando a saturação atrás da frente de injeção segundo o modelo de distribuição de fluido uniforme (Packwood, 1997). 
Figura 2.14 - Esquerda: Esquema representando a rocha com saturação heterogênea ou patchy. Direita: detalhe de cada heterogeneidade (Akbar et al, 2001).

Figura 2.15 - Esquema mostrando a saturação atrás da frente de injeção segundo o modelo de distribuição de fluido heterogênea (Packwood, 1997).

Figura 2.16 - Esquema da metodologia proposta por Sengupta (2000). 55 Figura 2.17 - Injeção de água: velocidade vs. saturação de água para diferentes saturações de óleo irredutível; a) $S_{o r}=20 \%$, b) $S_{o r}=30 \%$, c) $S_{o r}=0$ (Sengupta, 2000).

Figura 3.1 - Variação de $V_{p}$ em função de $S_{g}$ para os modelos homogêneo e patchy em arenito Beaver saturado com água e gás para $20^{\circ} \mathrm{C}$.

Figura 3.2 - Rocha Monterey saturada com óleo $50{ }^{\circ} \mathrm{API}$ e gás para $25^{\circ} \mathrm{C}$ e $125^{\circ} \mathrm{C}$. (a) Comparação entre os modelos homogêneo e patchy, (b) Diferença em porcentagem.

Figura 3.3 - Rocha Monterey saturada com óleo $50{ }^{\circ} \mathrm{API}$ e gás para $25^{\circ} \mathrm{C}$ e $125^{\circ} \mathrm{C}$. (a) Comparação entre os modelos homogêneo e patchy, (b) Diferença em porcentagem.

Figura 3.4 - Rocha Monterey saturada com óleo de $10{ }^{\circ} \mathrm{API}$ e gás para $25^{\circ} \mathrm{C}$ e $125^{\circ} \mathrm{C}$. (a) Comparação entre os modelos homogêneo e patchy, (b) Diferença em porcentagem.

Figura 3.5 - Comparação entre os modelos na rocha Monterey $\left(125^{\circ} \mathrm{C}\right)$ saturada com diferentes tipos de óleo e gás para $125^{\circ} \mathrm{C}$.

Figura 3.6 - Influência do tipo de fluido e da temperatura na heterogeneidade da saturação.

Figura 3.7 - Saturação de mistura água-gás em areia limpa para $P_{p}=10 \mathrm{MPa}$ e $P_{p}=30 \mathrm{MPa}$, e $\sigma^{\prime}=10 \mathrm{MPa}$. (a) Modelo homogêneo versus patchy, (b) diferença em porcentagem.

Figura 3.8 - Comparação entre os modelos aplicados em areia limpa ( $\phi=36 \%$ ) e areia argilosa ( $\phi=024 \%$ ). (a) Modelo homogêneo versus patchy, (b) diferença em porcentagem. 
Figura 3.9 - Modelo homogêneo versus patchy em arenito Fontainebleu saturado com óleo e gás para $5 \mathrm{MPa}$ de tensão efetiva. 70

Figura 4.1 - Modelo de simulação de fluxo 2D. 74

Figura 4.2 - Modelo de permeabilidade log-normal com $D P=0,63$, $L_{x}=4, L_{y}=0,5$.

Figura 4.3 - Módulo bulk do gás em função da temperatura e da pressão (Batzle \& Wang, 1992).

Figura 4.4 - Módulo bulk do óleo em função da temperatura e da pressão (Batzle \& Wang, 1992).

Figura 4.5 - Seções de saturação de gás para o cenário de injeção de gás leve em reservatório saturado com óleo de $25{ }^{\circ} \mathrm{API}$. De cima para baixo: 100, 500 e 1000 dias.

Figura 4.6 - Seções de saturação de gás para o cenário de injeção de gás pesado em reservatório saturado com óleo de $25{ }^{\circ} \mathrm{API}$. De cima para baixo: 100, 500 e 1000 dias.

Figura 4.7 - Seções de saturação de gás para o cenário de injeção de gás leve em reservatório saturado com óleo de $20{ }^{\circ} \mathrm{API}$. De cima para baixo: 100,500 e 1000 dias.

Figura 4.8 - Seções de saturação de gás para o cenário de injeção de gás pesado em reservatório saturado com óleo de $20{ }^{\circ} \mathrm{API}$. De cima para baixo: 100, 500 e 1000 dias.

Figura 4.9 - Seções de pressão do reservatório para caso de injeção de gás leve em reservatório saturado com óleo de $25{ }^{\circ} \mathrm{API}$. De cima para baixo: 100, 500 e 1000 dias.

Figura 4.10 - Seções de pressão do reservatório para caso de injeção de gás pesado em reservatório saturado com óleo $25^{\circ} \mathrm{API}$. De cima para baixo: 100, 500 e 1000 dias.

Figura 4.11 - Seções de pressão do reservatório para caso de injeção de gás leve em reservatório saturado com óleo de $20{ }^{\circ} \mathrm{API}$. De cima para baixo: 100, 500 e 1000 dias.

Figura 4.12 - Seções de pressão do reservatório para caso de injeção de gás pesado em reservatório saturado com óleo $20{ }^{\circ} \mathrm{API}$. De cima para baixo: 100, 500 e 1000 dias. 
Figura 5.1 - Escala sísmica versus escala de simulação de fluxo.

Figura 5.2 - Mapas de saturação de gás para o cenário de injeção de gás leve em reservatório saturado com óleo de $25{ }^{\circ} \mathrm{API}$. De cima para baixo: 100, 500 e 1000 dias.

Figura 5.3 - Seções de saturação de gás para o cenário de injeção de gás pesado em reservatório saturado com óleo de $25{ }^{\circ} \mathrm{API}$. De cima para baixo: 100, 500 e 1000 dias.

Figura 5.4 - Seções de saturação de gás para o cenário de injeção de gás leve em reservatório saturado com óleo de $20{ }^{\circ} \mathrm{API}$. De cima para baixo: 100, 500 e 1000 dias.

Figura 5.5 - Seções de saturação de gás para o cenário de injeção de gás pesado em reservatório saturado com óleo de $20{ }^{\circ} \mathrm{API}$. De cima para baixo: 100, 500 e 1000 dias.

Figura 5.6 - Variação de $V_{p}^{\text {homogêneo }}, V_{p}^{\text {patchy }}$ e $V_{p}^{\text {upsc }}$ (Gassmann) com a saturação de gás para 500 dias, para o cenário de injeção de gás leve em reservatório saturado com óleo de $25{ }^{\circ} \mathrm{API}$ para (a) $\phi=21 \%$, e (b) $\phi=30 \%$.

Figura 5.7 - Variação de $V_{p}^{\text {homogêneo }}, V_{p}^{\text {patchy }}$ e $V_{p}^{\text {upsc }}$ (Gassmann) com a saturação de gás para 500 dias, para o cenário de injeção de gás pesado em reservatório saturado com óleo de $25{ }^{\circ} \mathrm{API}$ para (a) $\phi=21 \%$, e b) $\phi=30 \%$.

Figura $5.8-V_{p}^{\text {upsc }}$ obtida através do modelo de M\&J para 500 dias, no cenário de injeção de gás leve em reservatório saturado com óleo de $25{ }^{\circ} \mathrm{API}$.

Figura 5.9 - Comparação entre $V_{p}^{u p s c}$ de Gassmann e $V_{p}^{u p s c}$ de M\&J para $\phi=30 \%$, para o cenário de injeção de gás pesado em reservatório saturado com óleo de $20{ }^{\circ} \mathrm{API}$.

Figura 5.10 - Exemplo de meio poroso (a) com baixa tortuosidade, (b) alta tortuosidade.

Figura 5.11 - Campos de velocidade compressional, $V_{p}$, obtidos usando Gassmann para injeção de gás leve em reservatório saturado com óleo de $25{ }^{\circ} \mathrm{API}, \phi=30 \%$. 
Figura 5.12 - Campos de $V_{p}$ obtidos com o modelo de M\&J para injeção de gás leve em reservatório saturado com óleo de $25{ }^{\circ} \mathrm{API}$, $\phi=30 \%, a=3$.

Figura 5.13 - Campos de $V_{p}$ obtidos com o modelo de Gassmann para injeção de gás pesado em reservatório saturado com óleo de $25^{\circ} \mathrm{API}$, $\phi=30 \%$.

Figura 5.14 - Campos de $V_{p}$ obtidos com o modelo de M\&J para injeção de gás pesado em reservatório saturado com óleo de $25{ }^{\circ} \mathrm{API}, \phi=30 \%, a=3$.

Figura 5.15 - Campos de $V_{p}$ obtidos através do modelo de Gassmann para injeção de gás leve em reservatório saturado com óleo de $20{ }^{\circ} \mathrm{API}$, $\phi=30 \%$.

Figura 5.16 - Campos de $V_{p}$ obtidos através do modelo de M\&J para injeção de gás leve em reservatório saturado com óleo de $20{ }^{\circ} \mathrm{API}$, $\phi=30 \%, a=3$.

Figura 5.17 - Campos de $V_{p}$ obtidos através do modelo de Gassmann para injeção de gás pesado em reservatório saturado com óleo de 20 ${ }^{\circ} \mathrm{API}, \phi=30 \%$.

Figura 5.18 - Campos de $V_{p}$ obtidos através do modelo de M\&J para injeção de gás pesado em reservatório saturado com óleo de $20{ }^{\circ} \mathrm{API}$, $\phi=30 \%, a=3$.

Figura 5.19 - Mapas de mudança de impedância acústica obtidos através do modelo de Gassmann para injeção de gás leve em reservatório saturado com óleo de $25{ }^{\circ} \mathrm{API}, \phi=30 \%$.

Figura 5.20 - Mapas de mudança de impedância acústica obtidos através do modelo de M\&J para injeção de gás leve em reservatório saturado com óleo de $25{ }^{\circ} \mathrm{API}, \phi=30 \%, a=3$.

Figura 5.21 - Mapas de mudança de impedância acústica obtidos através do modelo de Gassmann. Injeção de gás pesado em reservatório saturado com óleo de $25{ }^{\circ} \mathrm{API}, \phi=30 \%$.

Figura 5.22 - Mapas de mudança de impedância acústica obtidos através do modelo de $M \& J$, injeção de gás pesado em reservatório saturado com óleo de $25{ }^{\circ} \mathrm{API}, \phi=30 \%, a=3$. 
Figura 5.23 - Mapas de mudança de impedância acústica obtidos através do modelo de Gassmann para injeção de gás leve em reservatório saturado com óleo $20{ }^{\circ} \mathrm{API}, \phi=30 \%$.

Figura 5.24 - Mapas de mudança de impedância acústica obtidos através do modelo de $M \& J$ para injeção de gás leve em reservatório saturado com óleo $20^{\circ} \mathrm{API}, \phi=30 \%, a=3$.

Figura 5.25 - Mapas de mudança de impedância acústica obtidos através do modelo de Gassmann. Injeção de gás pesado em reservatório saturado com óleo de $20{ }^{\circ} \mathrm{API}, \phi=30 \%$.

Figura 5.26 - Mapas de mudança de impedância acústica obtidos através do modelo de $\mathrm{M \& J}$, injeção de gás pesado em reservatório saturado com óleo de $20{ }^{\circ} \mathrm{API}, \phi=30 \%, a=3$.

Figura 5.27 - Esquema da modelagem e cálculo da diferença.

Figura 5.28 - Geometria de aquisição utilizada na modelagem sísmica.

Figura 5.29 - Exemplo de sismograma para a) $t=0$ e b) $t=500$ dias. 109 Figura 5.30 - Exemplo da seção sísmica da diferença mostrando a frente de injeção no reservatório.

Figura 5.31 - Efeito time-lapse: injeção de gás leve em cenário com óleo de $25{ }^{\circ} \mathrm{API}, \phi=30 \%$ e tortuosidade (a) igual a 2, 2,5 e 3.

Figura 5.32 - Efeito time-lapse: injeção de gás leve em cenário com óleo de $25{ }^{\circ} \mathrm{API}, \phi=21 \%$ e tortuosidade (a) igual a 2, 2,5 e 3 . .

Figura 5.33 - Efeito time-lapse: injeção de gás pesado em cenário com óleo de $25^{\circ} \mathrm{API}, \phi=30 \%$ e tortuosidade (a) igual a 2, 2,5 e 3 ..

Figura 5.34 - Efeito time-lapse: injeção de gás pesado em cenário com óleo de $25{ }^{\circ} \mathrm{API}, \phi=21 \%$ e tortuosidade $(a)$ igual a 2, 2,5 e 3 . .

Figura 5.35 - Efeito time-lapse: injeção de gás leve em cenário com óleo de $20^{\circ} \mathrm{API}, \phi=30 \%$ e tortuosidade (a) igual a 2, 2,5 e 3.

Figura 5.36 - Efeito time-lapse: injeção de gás leve em cenário com óleo de $20^{\circ} \mathrm{API}, \phi=21 \%$ e tortuosidade (a) igual a 2, 2,5 e 3. Figura 5.37 - Efeito time-lapse: injeção de gás pesado em cenário com óleo de $20^{\circ} \mathrm{API}, \phi=30 \%$ e tortuosidade (a) igual a 2, 2,5 e 3. 
Figura 5.38 - Efeito time-lapse: injeção de gás pesado em cenário com óleo de $20^{\circ} \mathrm{API}, \phi=21 \%$ e tortuosidade (a) igual a 2, 2,5 e 3.

Figura 5.39 - Efeito time-lapse: injeção de gás leve em cenário com óleo de $25^{\circ} \mathrm{API}, \phi=30 \%$ e tortuosidade (a) igual a 2, 2,5 e 3 para $t=1000$ dias.

Figura A.1 - Interface UTCHEM. 


\section{Lista de tabelas}

Tabela 3.1 - Propriedades das rochas utilizadas

Tabela 3.2 - Variação dos parâmetros $K_{\text {óleo }}, K_{\text {sato }}, \rho_{\text {Bsato }}$ e seu

efeito em $V_{p}$.

71

Tabela 4.1 - Propriedades dos gases injetados no reservatório. 77

Tabela 4.2 - Propriedades do óleo no reservatório. 78

Tabela 4.3 - Cenários de injeção de gás simulados. 


\section{Lista de símbolos}

$a$

C

$D P$

$D$

$D_{p}$

$f_{c}$

$f_{n}$

$I$

$k$

$K_{d}$

$K_{d n r}$

$K_{f l}$

$K_{g}$

$K_{\text {het }}$

$K_{n}$

$K_{\text {nsat }}$

$K_{o}$

$K_{\text {oleo }}$

$K_{o r}$

$K_{\text {sat }}$

$K_{\text {sato }}$

$K_{\text {satO }}$
Tortuosidade (adimensional)

Volume de argila (\%)

Coeficiente de Dykstra-Parsons (adimensional)

Difusividade hidráulica $\left(\mathrm{m}^{2} / \mathrm{s}\right)$

Fator de pressão (adimensional)

Freqüência crítica $(\mathrm{Hz})$

Fração volumétrica do constituinte mineral

Impedância acústica (ohm)

Permeabilidade absoluta da rocha (mD)

Módulo bulk da rocha seca (MPa)

Módulo bulk da matriz não relaxada (MPa)

Módulo bulk do fluido (MPa)

Módulo bulk do gás (MPa)

Módulo bulk da rocha saturada com fluido distribuído de forma

heterogênea (MPa)

Módulo bulk do constituinte mineral

Módulo bulk da rocha saturada com a mistura de fluido,

modificado pela $S_{\text {or }}(\mathrm{MPa})$

Módulo bulk do mineral (MPa)

Módulo bulk do óleo (MPa)

Módulo bulk da rocha saturada, modificado pela $S_{\text {or }}(\mathrm{MPa})$

Módulo bulk da rocha saturada com o novo fluido (MPa)

Módulo bulk da rocha saturada com o fluido inicial (MPa)

Módulo bulk da rocha saturada com óleo (MPa) 


\begin{tabular}{|c|c|}
\hline$K_{w}$ & Módulo bulk da água (MPa) \\
\hline$L_{c}$ & Escala de difusão crítica (m) \\
\hline$L_{x}$ & Correlação espacial na direção $x$ (adimensional) \\
\hline$L_{y}$ & Correlação espacial na direção y (adimensional) \\
\hline$M$ & Razão de mobilidade (adimensional) \\
\hline$P_{p}$ & Pressão de poros (MPa) \\
\hline$S_{n}^{u p s c}$ & Saturação das diferentes fases na escala sísmica (\%) \\
\hline$S_{\text {or }}$ & Saturação de óleo residual (\%) \\
\hline$S_{w}$ & Saturação de água (\%) \\
\hline$V_{p}$ & Velocidade compressional (m/s) \\
\hline$V_{p}^{\text {homogêneo }}$ & $\begin{array}{l}\text { Velocidade compressional calculada com o modelo de saturação } \\
\text { homogênea }(\mathrm{m} / \mathrm{s})\end{array}$ \\
\hline$V_{p}^{\text {patchy }}$ & $\begin{array}{l}\text { Velocidade compressional calculada com o modelo de saturação } \\
\text { heterogênea }(\mathrm{m} / \mathrm{s})\end{array}$ \\
\hline$V_{p}^{\text {upsc }}$ & Velocidade compressional na escala sísmica (m/s) \\
\hline$V_{p 0}$ & $\begin{array}{l}\text { Velocidade compressional da rocha saturada com fluido inicial } \\
(\mathrm{m} / \mathrm{s})\end{array}$ \\
\hline$V_{s}$ & Velocidade cisalhante $(\mathrm{m} / \mathrm{s})$ \\
\hline$V_{s 0}$ & Velocidade cisalhante da rocha saturada com fluido inicial (m/s) \\
\hline$\alpha$ & Razão de achatamento dos poros (adimensional) \\
\hline$\Delta I$ & Variação da impedância acústica (\%) \\
\hline$\Delta K_{\text {rel }}$ & $\begin{array}{l}\text { Variação do módulo bulk da rocha saturada segundo as fissuras } \\
\text { presentes no meio (MPa) }\end{array}$ \\
\hline$\phi$ & Porosidade (\%) \\
\hline$\phi_{\text {equivK }}$ & $\begin{array}{l}\text { Porosidade compressível ou “compliante”associada à variação do } \\
\text { módulo bulk com a pressão (\%) }\end{array}$ \\
\hline
\end{tabular}




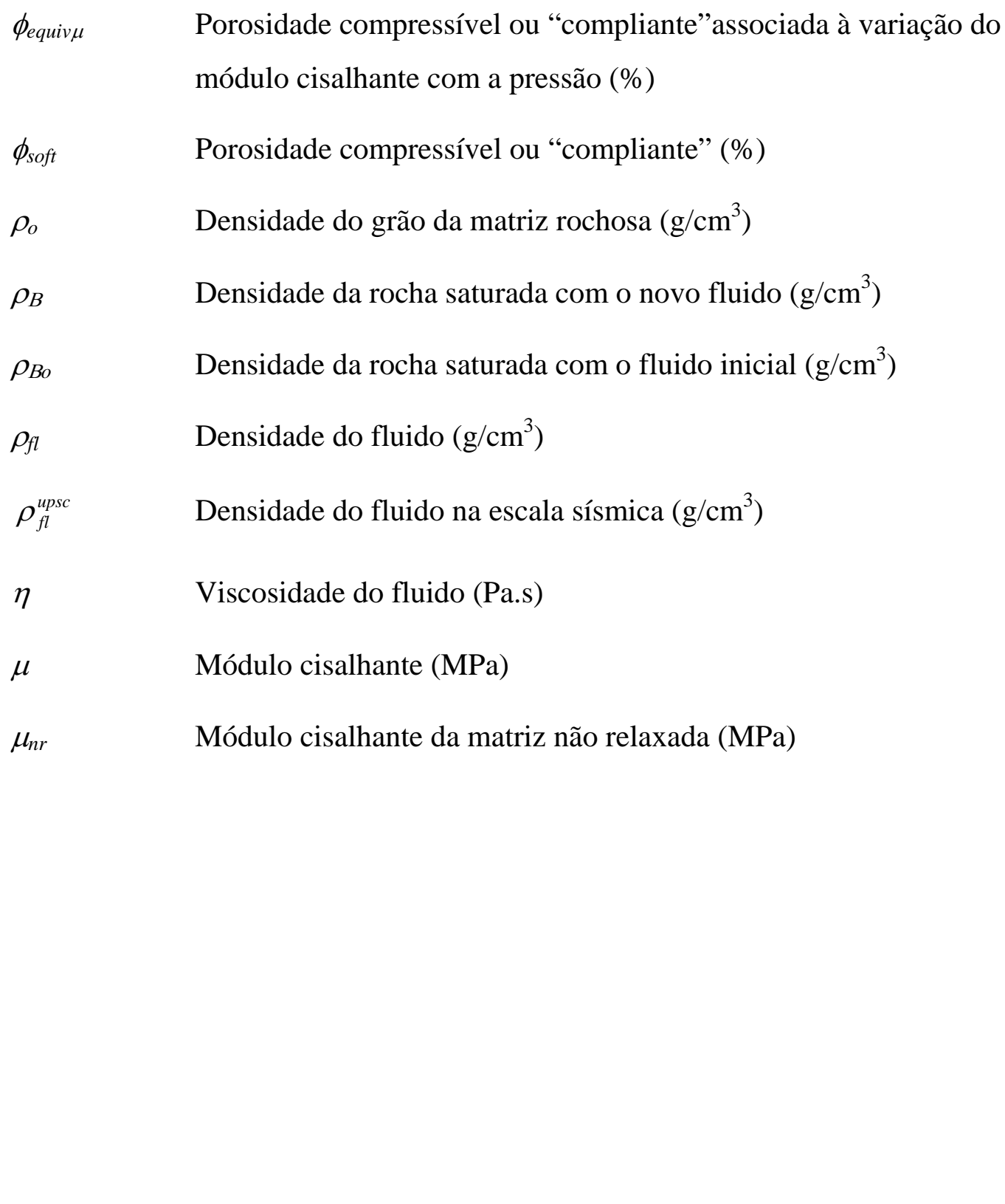


“...Y uno aprende a construir todos sus caminos en el hoy, porque el terreno de mañana es demasiado inseguro para planes... y los futuros tienen una forma de caerse en la mitad.

$Y$ después de un tiempo uno aprende que si es demasiado, hasta el calor del sol quema. Así que uno planta su propio jardín y decora su propia alma, en lugar de esperar a que alguien le traiga flores.

$Y$ uno aprende que realmente puede aguantar, que uno realmente es fuerte, que uno realmente vale, y uno aprende y aprende... y con cada día uno aprende..." 